\title{
Convective mechanism of amplification and structuring of magnetic fields
}

\author{
A. V. Getling, ${ }^{1}$ V. V. Kolmychkov ${ }^{2}$ and O. S. Mazhorova ${ }^{2}$ \\ ${ }^{1}$ Institute of Nuclear Physics, Lomonosov Moscow State University, Moscow, Russia, \\ email: A.Getling@mail.ru \\ ${ }^{2}$ Keldysh Institute of Applied Mathematics, Moscow, Russia, \\ email: ksv@keldysh.ru, olgamazhor@mail.ru
}

\begin{abstract}
Magnetoconvection in a horizontal layer of incompressible fluid is simulated numerically. The initial magnetic field is assumed to be uniform and horizontal. The interaction of quasi-ordered cellular convection with the magnetic field is shown to be able to produce bipolar (and also diverse more complex) configurations of a substantially amplified magnetic field. The operation of this mechanism, which can be regarded as a modification of the mechanism suggested by Tverskoi (1966), is controlled by the very topology of the cellular flow, should be manifest on various spatial scales, and does not require strong initial fields. Magnetic configurations develop both in the central parts of convection cells, where circulatory fluid motion "winds" magnetic field lines, and in the network formed by their peripheral regions due to the "sweeping" of magnetic field lines.
\end{abstract}

Keywords. Sun: magnetic fields, convection

\section{Introduction}

No unified physical explanation has been given as yet to the processes responsible for the formation of sunspot magnetic fields and small-scale magnetic elements. On the one hand, the development of the magnetic field of a bipolar sunspot group is traditionally attributed to the rising-tube mechanism. Specifically, it is assumed that some processes amplify the magnetic field in a flux tube lying at some depth below the photosphere and ultimately make it strong enough to produce a magnetic-buoyancy force sufficient for an $\Omega$-shaped loop of the tube to rise and emerge to the near-photospheric layers. The emerging segment of the tube intersects the photosphere at two sites, and two spots of opposite magnetic polarities develop precisely at these sites (Parker 1955). This view can, in particular, account for some global regularities of solar activity such as the Hale law of magnetic polarities. On the other hand, small-scale magnetic elements, which typically originate in the network formed by the lanes between convection cells on varying scales, are obviously not related to rising tubes. The formation of such elements is traditionally attributed to the sweeping of magnetic field lines by diverging convective motions.

In our opinion, the rising-tube model can hardly offer an adequate representation of reality. First of all, if it is adopted, one has to account for the origin of the strong magnetic field in the tube; to this end, some additional, fairly artificial assumptions need to be introduced. Second, and especially important, is that the evolution pattern inferred from this model for local photospheric magnetic fields disagrees with the pattern actually observed on the Sun. Let us note some points of this disagreement.

- The observed developing magnetic fields "seep" through the photosphere without breaking down the existing supergranular velocity field, in contrast to what should be expected if a flux tube emerges. 
- According to the rising-tube model, a strong horizontal magnetic field should be observed on the scale of the entire active region before the origin of a sunspot group. Actually, however, a fairly complex distribution of horizontal magnetic fields is recorded.

- The observed spreading flows are locally associated with each developing magnetic island rather than the entire complex, as could be expected in the case of the emergence of a tube. In particular, such small-scale flows can clearly be seen in the well-known "trilobite" magnetogram obtained on Hinode (2007).

- The presence of "parasitic" polarities within the area filled with a predominant magnetic polarity is not accounted for by the model.

- The coexistence of differently directed vertical velocities inside the regions of a given magnetic polarity appears to be inconsistent with the rising-tube model.

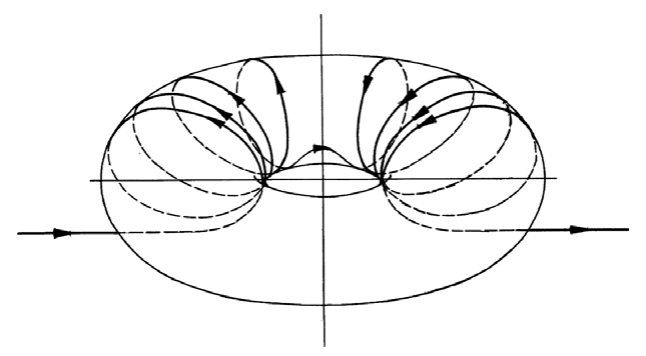

Figure 1. Tverskoi's toroidal eddy winding a magnetic field line.

An alternative possibility related to the winding of magnetic field lines by circulatory fluid motion was suggested by Tverskoi (1966). He considered a simple kinematic model describing the formation of a magnetic bipole by a toroidal eddy in perfectly conducting fluid. The action of Tverskoi's mechanism is illustrated in Fig. 1. The fluid particles move in circular trajectories, and the circles of a given radius form a toroidal surface. The magnetic field lines, which are initially straight and horizontal, are wound by the fluid motion around the tori and form two flux concentrations, with oppositely directed magnetic fields, in the central part of the eddy. Tverskoi's prediction was later qualitatively confirmed by nonlinear numerical simulations, which were carried out for the cases where the flow corresponds to a pattern of hexagonal, Bénard-type cells and is strongly stabilised by the conditions of periodicity in the horizontal directions (Getling 2001, Dobler \& Getling 2004). As can be seen from Fig. 2, a bipolar magnetic structure similar to that found by Tverskoi develops in each convection cell. In addition, the vertical component of the magnetic field is enhanced in the contact zones between the hexagonal cells, i.e., in the intercellular lanes.

Tverskoi considered the toroidal eddy to be a schematic representation of a convection cell. In essence, he demonstrated that the topology of the flow is most important in terms of the MHD effects of convection. He conjectured that supergranular convection cells, interacting with the latitudinal component of the global magnetic field $\dagger$ in nearly the same way as such eddies interact with the horizontal initial field, could be producers of the magnetic fields of bipolar sunspot groups. If so, Tverskoi's model appears to be as successful as the rising-tube model in terms of agreement with the Hale polarity law and other global properties of solar activity.

Since well-developed sunspot groups are typically much larger than supergranules, it may seem natural to modify Tverskoi's interpretation in some way. In particular, if especially large and energetic cells sometimes originate in subphotospheric layers then, interacting with the global latitudinal field, they could occasionally give rise to the magnetic fields of bipolar groups, in contrast to the ubiquitous "normal" supergranules, which

$\dagger$ Since we discuss here the properties of toroidal eddies, we do not use the commonly accepted term toroidal magnetic field to avoid terminological confusions. 

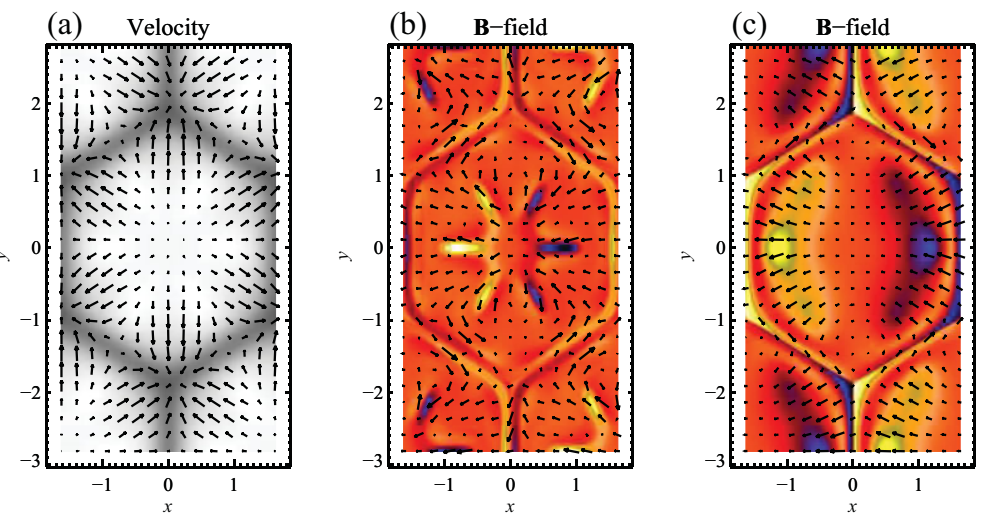

Figure 2. (a) Velocity field and (b, c) magnetic field (light for positive and dark for negative values of the $z$ components of the vectors, arrows for their horizontal components) obtained in simulations with the horizontal-periodicity conditions (Dobler \& Getling 2004). The plots refer to horizontal sections of the computation box that are located near the top surface (a, c) and near the midplane of the layer (b).

could be expected to usually produce smaller-scale fields. On the other hand, the convective mechanism can act on smaller spatial scales, being responsible for the development of various local magnetic features. It can also act in parallel with the process of magneticfield-line sweeping.

If such a convective mechanism is regarded as a regular producer of sunspot magnetic fields, we have to note that, in contrast to the rising-tube mechanism, it is free of some disagreements with observations. Let us list the points at which the convective mechanism is better compatible with observational data.

- The consistency of the developing magnetic field with the convective velocity field is an inherent property of the mechanism.

- The amplified magnetic field should largely be collinear with the streamlines, and a strong horizontal field does not connect different polarities.

- Spreading flows are actually associated with developing magnetic islands rather than the entire complex.

- Diverse complex patterns with mixed polarities can be accounted for in a natural way by the presence of a fine structure of the convective flow.

- Although the question of the scale of the produced bipolar fields remains disputable, it is worth noting that the convective mechanism can in principle operate on various spatial scales, being controlled solely by the topology of the flow.

Indeed, some class of possible manifestations of the convective mechanism may be related to magnetic fields observed on smaller scales. As observations show, magnetic elements frequently develop in the inner parts of supergranules (Title 2006), and this process cannot be associated with the sweeping of magnetic field lines but can be attributed to field-line winding.

According to the current nomenclature, Tverskoi's model can be classified as a localdynamo model. Likely, this was the earliest model of the sort. However, while local dynamos are predominantly regarded as generators of small-scale, highly intermittent magnetic fields (Cattaneo 1999), the convective mechanism discussed here, as already noted, can act in a wide range of scales. 


\section{Formulation of the problem}

We simulate magnetoconvection in a layer of incompressible fluid heated from below, and our computation box measures $8 \times 8 \times 1$ in units of the layer thickness $H$. As the thermal boundary conditions, we specify a fixed temperature at the bottom layer boundary and zero heat flux through the upper boundary and sidewalls. Thus, the stabilising effect of the horizontal-periodicity conditions is now eliminated, and more freedom is offered to the flow.

For the simulated flow to form relatively stable three-dimensional cells resembling those observed on the Sun, we additionally introduce uniformly distributed volume heat sinks, which produce a parabolic static temperature profile. In a nondimensional form, it is given by the equation $T=1-2 z+z^{2}$ (see Fig. 3 ). No-slip conditions are assumed at all boundaries of the region, and the initial magnetic field $\mathbf{B}_{0}$ is uniform and directed along the horizontal $x$ axis. The magnetic field remains constant at the sidewalls in the course of flow evolution.

The physical parameters of the problem are the quantities

$$
\mathrm{Ra}=\frac{\alpha g \Delta T H^{3}}{\nu \chi}, \quad \operatorname{Pr}=\frac{\nu}{\chi}, \quad \operatorname{Pr}_{\mathrm{m}}=\frac{4 \pi \sigma \nu}{c^{2}}, \quad \mathrm{Ha}=\frac{B_{0} H}{c} \sqrt{\frac{\sigma}{\rho \nu}},
$$

known as the Rayleigh number, the hydrodynamic and the magnetic Prandtl number, and the Hartmann number, respectively, and also the nondimensional heat-source density (negative in our case)

$$
q=\frac{Q}{c_{p} \rho \Delta T} \frac{\nu}{H^{2}}
$$

set equal to $(-2 / \mathrm{Pr})$. Here, $\alpha$ is the volumetric expansion coefficient of the fluid, $g$ is the gravitational acceleration, $\Delta T$ is the temperature difference between the bottom and top layer surfaces (it can be calculated, once both the bottom-surface temperature and heat-source density are specified), $\nu$ and $\chi$ are the kinematic viscosity and thermal diffusivity of the fluid, $\sigma$ is its electrical conductivity, $\rho$ is its density, $Q$ is the dimensional heat-source density and $c_{p}$ is the specific heat at constant pressure.

To solve the Navier-Stokes equation combined with the equations of electromagnetic induction, heat transfer, and continuity, we use the SIMPLE algorithm [Semi-Implicit Method for Pressure-Linked Equations, see Fletcher

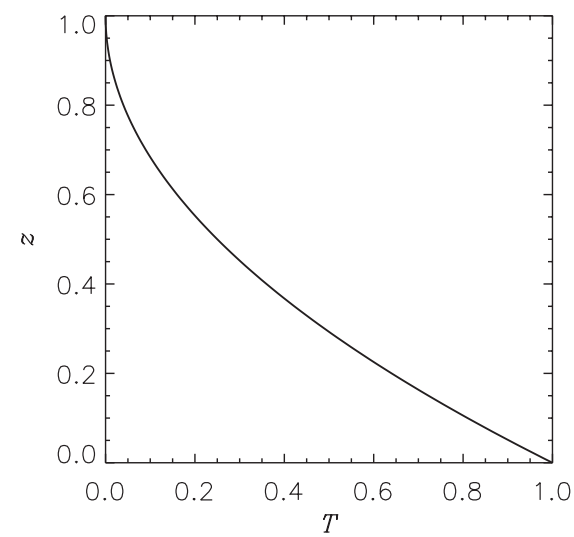

Figure 3. Static temperature profile. (1991)] modified by Kolmychkov, Mazhorova \& Popov (2006a, 2006b).

For the conditions of our problem, the critical Rayleigh number $\mathrm{Ra}_{\mathrm{c}}$ was not known from previous analyses, and we determined it by varying the parameters in our computations.

\section{Results}

We start our simulations with introducing a random field of thermal perturbations. At the initial evolutionary stage, a system of cells develops in the form of irregular polygons. Locally amplified vertical magnetic fields emerge both in the intercellular network and 
in the inner parts of cells (Fig. 4, left). It is remarkable that the magnetic intracellular magnetic elements are bipolar in their structure, in agreement with the prediction by Tverskoi (1966). In their further evolution, many of bipolar structures change into unipolar ones, reaching high strengths in many cases (a few hundreds of $B_{0}$ ) rather than weakening (the latter could be expected if they mutually annihilated); see Fig. 4, right. In some cases, a dissymmetry of bipolar elements can be noted: one of the polarities acquires a diffuse appearance, while the other remains compact. The visualisation of the motion of tracers, or corks, demonstrates that the amplification of the magnetic field is not a transient process, and reciprocating cork motion is indicative of the circulation of fluid particles, which is a substantial element of the amplification mechanism.
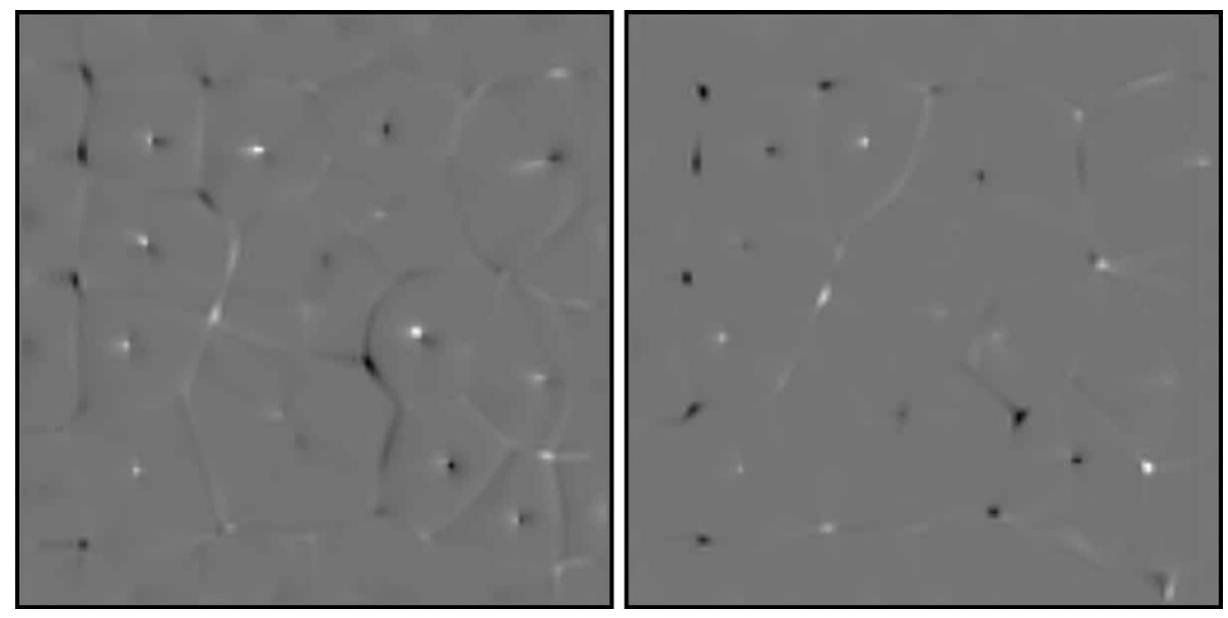

Figure 4. Maps of the vertical component of the magnetic fields obtained at $\mathrm{Ra} \approx 50 \mathrm{Ra}_{\mathrm{c}}, \mathrm{Pr}=$ $30, \operatorname{Pr}_{\mathrm{m}}=300, \mathrm{Ha}=0.01$. The left-hand and the right-hand plot correspond to different times.

For the run illustrated in Fig. 4, we assumed $\mathrm{Ra} \approx 50 \mathrm{Ra}_{\mathrm{c}}, \operatorname{Pr}=30, \mathrm{Pr}_{\mathrm{m}}=300$, $\mathrm{Ha}=0.01$. The computations were carried out until $t=26 t_{\nu}$, where $t_{\nu}=H^{2} / \nu$ is the time of viscous dissipation on the scale $H$. The maximum magnetic-field strengths achieved by this time were about $600 B_{0}$ at the bottom and about $460 B_{0}$ at the top of the layer. In the cases run, the values at the bottom systematically exceed the values at the top, reaching $2500 B_{0}$ in a run with $\mathrm{Ra} \approx 100 \mathrm{Ra}_{\mathrm{c}}$. Likely, this excess should be attributed to the phenomenon of topological magnetic-flux pumping (Drobyshevski \& Yuferev 1974).

Thus, this model of the convective mechanism, although it may appear to be oversimplified, demonstrates nevertheless the ability of quasi-ordered cellular convection to produce strongly amplified magnetic fields similar to those observed in the photosphere. Obviously, the replacement of the uniform horizontal initial magnetic field with a field of a more complex structure would necessarily result in the formation of a variety of configurations of the amplified field, which should generally be multipolar. Some role in the development of magnetic structure can be played by magnetic buoyancy; discussing its effects goes beyond our consideration.

The magnetic structures can develop both in the intercellular network, due to the sweeping of magnetic field lines, and near the centres of convection cells, where the field lines are wound by circulatory fluid motion. The action of this mechanism is controlled by the very topology of the cellular flow, can be manifest on various spatial scales, and does not require strong initial magnetic fields. 


\section{Conclusion}

To summarise, we note the following:

- Local concentrations of magnetic field can develop both in the intercellular network and inside convection cells. The second scenario agrees with Tverskoi's model, and bipolar configurations form an important class of magnetic structures formed in this way.

- In many cases, the components of a bipolar structure merge with each other without annihilation, giving rise to unipolar magnetic elements.

- The topology of the flow plays a fundamental role in the operation of the mechanism of magnetic-filed amplification and structuring, and it can be expected that the regularities of the process should similarly manifest themselves on different spatial scales.

\section{Acknowledgement}

This work was supported by the Russian Foundation for Basic Research, project 12-02-00792-a.

\section{References}

Cattaneo, F. 1999, ApJ, 515, L39.

Dobler, W. \& Getling, A. V. 2004, in: A. V. Stepanov, E. E. Benevolenskaya \& A. G. Kosovichev (eds.), Multi-Wavelength Investigations of Solar Activity, Proc. IAU Symposium No. 223 (Cambridge: CUP), p. 239

Drobyshevski, E. M. \& Yuferev, V. S. 1974, J. Fluid Mech., 65, 33

Fletcher, C. A. J. 1991, Computational Techniques for Fluid Dynamics, 2nd Ed., (Berlin: Springer)

Getling, A. V. 2001, AZh, 78, 661 (Astron. Rep., 45, 569, 2001)

Hinode 2007, http://science.nasa.gov/science-news/science-at-nasa/2007/18sep_trilobite/

Kolmychkov, V. V., Mazhorova, O. S., \& Popov, Yu.P. 2006a, Differential Equations, 42, 994

Kolmychkov, V. V., Mazhorova, O. S., \& Popov, Yu.P. 2006b, Math. Modelling Analysis, 11, 57 Parker, E.N. 1955 ApJ, 121, 491

Title, A. 2006, Lecture at the IAU XXVI General Assembly, Prague

Tverskoi, B. A. 1966, Geomagn. Aeron., 6, 11

\section{Discussion}

ABRAmEnKo: As far as I understand you, the emergence of unipoles was really observed in your simulations. If so, how do the bipoles change into unipoles?

Gething: At the moment, we cannot yet describe this process in detail. A plausible conjecture is that one polarity becomes "dissolved," while the other remains compact. 